\title{
Improving Sepsis Management in the Acute Admissions Unit
}

\author{
Laura Adcroft
}

Great Western Hospital

\begin{abstract}
Sepsis is a common condition with a major impact on healthcare resources and expenditure. We therefore wanted to investigate and improve how the acute admission unit (AAU) at the Great Western Hospital $(\mathrm{GWH})$ is managing patients who present directly to the unit with sepsis. In order to obtain this information, an audit was undertaken against the College of Emergency Medicine standards used by the emergency department within GWH and across the UK. Data was retrospectively collected for 30 patients with a diagnosis of severe sepsis or septic shock. The notes were scrutinized with regard to the implementation of College of Emergency Medicine standards for the management of sepsis. This meant that performance in the AAU was compared against the emergency department at GWH and national figures.
\end{abstract}

The data collected shows performance is below national standards with regard to documentation of high flow oxygen use (AAU: $24 \%$, ED 100\%; national median: 50\%; CEM standard 95\%), crystalloid fluid boluses (AAU: 52\%; ED: 90\%; national median: 83\%; CEM standard 100\%), lactate measurements (AAU: 66\%, ED: $93 \%$; national median: $80 \%$; CEM standard $95 \%$ ), and obtainment of blood cultures (AAU: 52\%; ED 73\%; national median: 77\%; CEM standard: $95 \%$ ). Only 3\% of patients received all six parts of the sepsis bundle.

Since auditing in 2012/2013 we have introduced a sepsis proforma based on a current proforma being used within Severn Deanery. This proforma uses the 'Sepsis Six' bundle appropriate to ward based care. We have raised awareness of sepsis implications and management through the creation of a 'sepsis working group' to educate both junior doctors and nurses. In turn, this has led to education through the use of posters, pocket reference cards, and teaching sessions. Re-audit shows significant improvement in administering all parts of the Sepsis Six bundle and an $8 \%$ improvement in patients receiving all six of the bundle.

\section{Problem}

In the acute admissions unit at Great Western Hospital, patients are admitted directly to the ward via GP referrals. As of last year we had no clear system for identifying patients suffering from sepsis, or a clear algorithm for treating them. They were therefore being coded incorrectly and not being identified early on to receive the Sepsis Six bundle. Awareness of identifying features of sepsis within nursing and medical staff was variable, and performance at administering oxygen, fluids, and antibiotics within a one hour time period was poor. Staff's documentation of taking lactate, urine output, and blood cultures was equally poor as demonstrated by an audit against the Royal College of Emergency Medicine standards for treatment of severe sepsis and septic shock.

\section{Background}

Sepsis is a common condition with a major impact on healthcare resources and expenditure. Defined as the presence (probable or documented) of infection together with systemic manifestations of infection or systemic inflammatory response syndrome (SIRS). Severe sepsis is defined as sepsis plus sepsis-induced organ dysfunction or tissue hypoperfusion. The implications of severe sepsis are a mortality rate of approximately $40-50 \%$ in the context of optimal management.[1] More detailed parameters of severe sepsis and septic shock are outlined in the methods section.

This life threatening condition undoubtedly requires appropriate management, and to have confidence in such treatment plans requires a fundamental basis upon which to focus.

The Surviving Sepsis Campaign was launched as a collaboration of three professional organizations at the European Society of Intensive Care Medicine's annual congress in Barcelona in 2002. It outlined six key areas or bundles in the initial work up for septic patients. These bundles are essentially a core set of recommendations, the majority of which are within the scope of most healthcare worker's practice while others require specialist skills. Some have argued that the latter tasks are difficult to achieve outside well resourced units and requires effective communication between point-of-access, admitting and critical care teams, with advice and clinical support from radiology, microbiology, and infection control personnel.[2]

\section{Baseline measurement}

We looked at 30 sets of casenotes obtained from patients admitted to the acute medical unit between 2012 to 2013. These were all patients fulfilling the criteria for a diagnosis of severe sepsis or septic shock. The inclusion criteria are listed below but included only those 'medically expected' patients. These were mainly GP referrals or admissions from clinic and thus had not been assessed by the accident and emergency doctors.

Case notes were obtained retrospectively via the audit department using codes for severe sepsis or septic shock. These were then screened to ensure eligibility as per inclusion criteria. In addition, 
BMJ Quality Improvement Reports

eligible patients were identified on the ward through the electronic patient admissions list. Filters for GP referral were used to identify eligible patients and then the reason for referral was used to identify patients with a potential diagnosis of sepsis. The auditors screened these notes to determine if patients fulfilled a diagnosis of severe sepsis or septic shock. Case notes were then compared against the College of Emergency Medicine standards for the treatment of severe sepsis.

\section{a. Inclusion criteria}

1. To fulfill the requirements for a diagnosis of severe sepsis or septic shock

2. To have been referred directly to AAU via the GP, hence 'medically expected'

3. All cases must be from the adult population (aged 18 years or more)

b. Exclusion criteria

1. Patients already assessed outside of AAU e.g referred from the Emergency Department

2. Neutropenic sepsis cases (as a proforma for management already exists at $\mathrm{GWH}$ )

Results:

Areas specifically of concern include:

Measurements and recordings:

Blood glucose (AAU Swindon: 62\%; ED Swindon: 100\%; National median: $81 \%$, standard 95\%)

High flow oxygen (AAU Swindon: 24\%; ED Swindon: 90\%; National median: 50\%, standard 95\%)

First fluid bolus (AAU Swindon: 52\%; ED Swindon: 90\%; National median: $83 \%$, standard 100\%)

Serum lactate (AAU Swindon: 66\%, ED Swindon: 93\%; National median: $80 \%$, standard $95 \%$ )

Blood cultures (AAU Swindon: 52\%; ED Swindon: 73\%; National median: $77 \%$, standard $95 \%$ ).

Patients receiving Sepsis Six bundles:

Six parts: $1(3 \%)$

Five parts: $3(10 \%)$

Four parts: $6(20 \%)$

Three parts: $13(44 \%)$

Two parts: $4(13 \%)$
One part: $3(10 \%)$

See supplementary file: ds3378.docx - "Results table 1"

\section{Design}

Our intervention was several fold and with reasons to support each. The first of these was the introduction of a sepsis proforma. This was placed in admission notes to allow the identification of potentially septic patients through easy visualisation of the criteria and management using the

Sepsis Six bundle. A similar proforma was being used to good effect elsewhere in the deanery, and with their permission we adopted and adapted this in order to incorporate it into the working proforma of the department. In keeping with this first intervention we used the simplified Sepsis Six bundle appropriate to ward based care. We felt it was important to use a validated tool which allowed for simple interpretation and use.

Our second major intervention was to raise awareness of sepsis implications and management through the creation of a junior doctor group to educate both junior doctors and nurses. By creating a working group we have been able to start an intervention which is now self-fulfilling. As junior doctors move frequently across jobs, these doctors can continue to hand over the reigns to the next in order to continue improving sepsis management through the years. Before this working group was formed, we implemented education through the use of posters and teaching sessions. This aimed to kick start people's awareness of the problem and educate doctors and nurses in the identification and management of sepsis. Other simple measures were introduced, including visual aids in order to maintain awareness. With the implementation of these changes we can then continue to monitor improvement in cycles through an already developed data collection tool.

\section{Strategy}

PDSA cycle 1: In the first PDSA cycle initial education and awareness talks were given to the AAU department, during which it was identified how we could improve our educational talks to ease understanding at future educational seminars. This included changing the way data was displayed to make it easier to interpret receiving initial views on how visual aids could be improved, and ways to set up and perpetuate working groups. The following cycles were then carried on by the sepsis working group.

PDSA cycle 2: The second cycle consisted of educational talks that were rolled out more widely across the hospital to junior doctors and nurses working in other departments. This was because they often worked across multiple sites including AAU, providing further feedback and improvements to be made. The second generation of visual aids was created, including sepsis posters and cards to carry on name badges.

PDSA cycle 3: In the third cycle it was identified that the time to carry out the Sepsis Six was restricted by collecting all of the 
necessary materials and knowledge of how to set up IV antibiotics by junior doctors. Therefore a sepsis trolley was introduced and initial planning for setting up educational sessions for junior doctors began.

PDSA cycle 4: A fourth cycle saw educational sessions for each set of junior doctors rotating through the department has been implemented through the sepsis working group. Through MDT sessions, which are consultant and nurse led, junior doctors are taught to set up IV antibiotics in the absence of a nurse available to administer them.

The ongoing identification and rewarding of staff members who have demonstrated excellence in the understanding, management, or teaching of sepsis interventions has been implemented in order to create an environment of positive reinforcement to tackling sepsis.

An audit of performance against CEM standards as well as use of the sepsis proforma and use of the Sepsis Six bundle when appropriate had commenced six months after the proforma and other practical changes were in place.

\section{Results}

After the intervention the sepsis working group audited the percentage of patients receiving the Sepsis Six Bundle one year on from the original baseline measurements.

There has been a positive shift in the percentage of patients receiving more elements of the sepsis six bundle than prior to interventions.

Patients receiving Sepsis Six bundles:

Six parts: $2014: 11 \%$ 2013: $3 \%$

Five parts: $2014: 47 \%$ 2013: $10 \%$

Four parts: 2014: 29\% 2013: $20 \%$

Three parts: 2014: $11 \%$ 2013: $44 \%$

Two parts: 2014: 0\% 2013: 13\%

One part: $2014: 0 \%$ 2013: $10 \%$

\section{Lessons and limitations}

Several lessons were learned along this journey and limitations occurred at most steps. The first of these was recording baselines measurements. This initially appeared to be a straightforward task which was soon complicated by identifying patients which fulfilled the criteria. Since severely unwell patients are often taken directly to the emergency department, the 'obvious' septic shock patients rarely come directly to AAU. Therefore, case notes were often not coded as "sepsis". This led to a slower process of looking at notes coded as infection and then determining if they were septic on arrival. This process has been sped up at re-audit as staff on posttake rounds have been asked to e-mail likely appropriate patient identifiers securely to the data collectors.

Another important limitation identified through one cycle was that timely management was reliant on more than education and visual aids. Allowing quick access to the vital equipment and ensuring all staff were competent in using the equipment available on the sepsis trolley should aid in improving time to treatment.

An important lesson learned in this project is the value of setting up a working group. This has allowed the interventions to remain sustained and evolve with the changing environment of the ward. Another important lesson learned is the importance of recognising achievements. Recognition and reward has proved an important part of the PDSA cycles to changing staff attitudes and morale towards identifying and managing sepsis.

\section{Conclusion}

By implementing multiple interventions to improve awareness and management of sepsis, we have achieved a definite change in attitude on the ward. Awareness of recognising and managing sepsis is anecdotally better. It is important to recognise that it would have been useful to test knowledge of this area before implementing changes through questionnaires to show definite improvement among stable staff and rotating junior doctors on the ward. The second audit has shown a slow but definite improvement in the percentage of the Sepsis Six bundle being received by patients. In turn this will have better outcomes for individuals who present to AAU with sepsis and also long-term economical benefits for the hospital.

\section{References}

1. Schmidt, G., Mandel, J. Evaluation and management of severe sepsis and septic shock in adults. Uptodate.com. 2013. Topic 1613 Version 30.0.

2. Daniels, R. Surviving the first hours in sepsis: getting the basics right (an intensivist's perspective). J Antimicrob Chemother. 2011;66 Suppl 2:i11-23.

\section{Declaration of interests}

Nothing to declare.

\section{Acknowledgements}

Agents for Change Conference 2014: Dr. Amanda Pegden, Richard Turck, Christopher Devine, Priya Rajyaguru. 